\title{
A Remark on the decay property for the Klein-Gordon equation in anti-de Sitter space time
}

Muhammet Yazici

Department of Mathematics, Faculty of Sciences, Karadeniz Technical University, Trabzon, Turkey

Received: 20 June 2017, Accepted: 5 August 2017

Published online: 8 November 2017.

Abstract: We consider the inital value problem for the Klein-Gordon equation in anti-de Sitter spacetime. We derive the pointwise decay estimate by using the fundamental solution to the linear Klein-Gordon equation in anti-de Sitter spacetime with source term.

Keywords: Anti-De Sitter spacetime, Klein-Gordon eqution, fundamental solution, pointwise estimate.

\section{Introduction}

In this article, we consider the decay estimate for the solution of the following Klein-Gordon equation in anti-de Sitter spacetime:

$$
\begin{aligned}
& \partial_{t}^{2} u-e^{2 t} \Delta u+M^{2} u=f(x, t), \quad(x, t) \in \mathbb{R}^{n} \times \mathbb{R}, \\
& \Phi(x, 0)=0, \quad \partial_{t} \Phi(x, 0)=0, \quad x \in \mathbb{R}^{n},
\end{aligned}
$$

where $f \in C^{\infty}\left(\mathbb{R}^{n+1}\right)$. The curved mass $M$ is defined as follows: $M^{2}:=m^{2}-n^{2} / 4$ where $m>0$ represents the physical mass. We briefly review how the equation in (1) is deduced. The line element in de Sitter spacetime is given by

$$
d s^{2}=-\left(1-\frac{r^{2}}{R^{2}}\right) d t^{2}+\left(1-\frac{r^{2}}{R^{2}}\right)^{-1} d r^{2}+r^{2}\left(d \theta^{2}+\sin ^{2} \theta d \phi^{2}\right)
$$

where $R$ is the radius of the universe. By using the Lemaitre-Robertson transformation in [6],

$$
r^{\prime}=\frac{r}{\sqrt{1-r^{2} / R^{2}}} e^{-t / R}, \quad t^{\prime}=t+\frac{R}{2} \ln \left(1-\frac{r^{2}}{R^{2}}\right), \quad \theta^{\prime}=\theta, \quad \phi^{\prime}=\phi
$$

the line element has the following form

$$
d s^{2}=-d t^{\prime 2}+e^{2 t^{\prime} / R}\left(d r^{\prime 2}+r^{\prime 2} d \theta^{\prime 2}+r^{\prime 2} \sin ^{2} \theta^{\prime} d \phi^{\prime 2}\right) .
$$

Changing the coordinate as

$$
t=t^{\prime}, \quad x_{1}=r^{\prime} \sin \theta^{\prime} \cos \phi^{\prime}, \quad x_{2}=r^{\prime} \sin \theta^{\prime} \sin \phi^{\prime}, \quad x_{3}=r^{\prime} \cos \theta^{\prime},
$$

we get

$$
d s^{2}=-d t^{2}+e^{2 H t}\left(d x_{1}^{2}+d x_{2}^{2}+d x_{3}^{2}\right)
$$


where we put $H=1 / R$. We may write the line element in the general spatial dimensions as

$$
d s^{2}=-d t^{2}+e^{2 H t}\left(d x_{1}^{2}+\ldots+d x_{n}^{2}\right) .
$$

For simplicity, we set $H=1$. Thus the corresponding metric is

$$
\left(g_{i k}\right)_{0 \leq i, k \leq n}:=\operatorname{diag}\left(-1, e^{2 t}, \ldots, e^{2 t}\right) .
$$

Let $g:=\operatorname{det}\left(g_{i k}\right)_{0 \leq i, k \leq n}$ and $\left(g^{i k}\right)_{0 \leq i, k \leq n}$ be the inverse matrix of $\left(g_{i k}\right)_{0 \leq i, k \leq n}$. Then the scalar field $\Phi$ in de Sitter spacetime is described by the following equation:

$$
\frac{1}{\sqrt{|g|}} \frac{\partial}{\partial x_{i}}\left(\sqrt{|g|} g^{i k} \frac{\partial \Phi}{\partial x_{k}}\right)=m^{2} \Phi+f
$$

where $x_{0}:=t$. More explicitly, we get the following equation

$$
\partial_{t}^{2} \Phi+n \Phi_{t}-e^{-2 t} \Delta \Phi+m^{2} \Phi=f \quad(x, t) \in \mathbb{R}^{n} \times \mathbb{R}
$$

If we introduce the new unknown function $u=e^{\frac{n}{2} t} \Phi$, the equation (5) takes the form of the linear Klein-Gordon equation in de Sitter spacetime

$$
\partial_{t}^{2} u-e^{-2 t} \Delta u+M^{2} u=f
$$

The time inversion transformation $t \rightarrow-t$ reduces the equation (6) to the equation in (1)

$$
\partial_{t}^{2} u-e^{2 t} \Delta u+M^{2} u=f
$$

that is regarded as the equation in anti-de Sitter space time.

In Minkowski spacetime, the initial value problem for the semilinear Klein-Gordon equation

$$
u_{t t}-\Delta u+m^{2} u=|u|^{\alpha} u
$$

has been extensively investigated. The existence of global weak solutions has been obtained by Jörgens [5], Pecher [7], Brenner [2], Ginibre and Velo [3,4]. In order that the total energy is well-defined in the energy space, one needs the assumption $\alpha<4 /(n-1)$.

Turning back to the initial value problem (1), the following theorem obtained by Galstian [9] states the estimate in the Sobolev space $H^{s}\left(\mathbb{R}^{n}\right)$.

Theorem 1. Let $u=u(x, t)$ be the solution of the initial value problem

$$
u_{t t}-e^{2 t} \Delta u+M^{2} u=f, \quad u(x, 0)=0, \quad u_{t}(x, 0)=0
$$

for $(x, t) \in \mathbb{R}^{n} \times(0, \infty)$, where $f \in C^{\infty}\left(\mathbb{R}^{n+1}\right)$. Let $l$ be a nonnegative integer, $m \geq n / 2$ and $n \geq 2$. Then there exists a constant $C>0$ such that

$$
\left\|(-\Delta)^{-s} u(., t)\right\|_{W^{l, q}\left(\mathbb{R}^{n}\right)} \leq C e^{t\left(2 s-n\left(\frac{1}{p}-\frac{1}{q}\right)\right)} \int_{0}^{t}\|f(., b)\|_{W^{l, p}\left(\mathbb{R}^{n}\right)}(1+t-b)^{1-\operatorname{sgn} M} d b
$$


for all $t>0$, provided that $s \geq 0,1<p \leq 2, \frac{1}{p}+\frac{1}{q}=1, \frac{1}{2}(n+1)\left(\frac{1}{p}-\frac{1}{q}\right) \leq 2 s \leq n\left(\frac{1}{p}-\frac{1}{q}\right)<2 s+1$. Here we have set $M=\sqrt{m^{2}-\frac{n^{2}}{4}}$.

The decay estimate is an important tool to prove the global existence for nonlinear partial differential equations. On the other hand, we are interested in the pointwise decay estimate for the solution of (1). The limiting case $q=\infty$ (i.e. $p=1)$ for the decay estimate is excluded in Theorem 1. We remark that the decay rate for the pointwise decay estimate is faster than the decay rate for the $L^{2}$ decay estimate. Therefore, by using the pointwise decay estimate, we prove the following theorem.

Theorem 2. Let $u=u(x, t)$ be the solution of the initial value problem

$$
u_{t t}-e^{2 t} \Delta u+M^{2} u=f, \quad u(x, 0)=0, \quad u_{t}(x, 0)=0
$$

for $(x, t) \in \mathbb{R}^{n} \times(0, \infty)$, where $f \in C^{\infty}\left(\mathbb{R}^{n+1}\right)$. Let $m \geq n / 2$ and $n \geq 2$. Then there exists a constant $C>0$ such that

$$
\|u(., t)\|_{L^{\infty}\left(\mathbb{R}^{n}\right)} \leq C \int_{0}^{t}\|f(., b)\|_{W^{[n / 2]+1,1}\left(\mathbb{R}^{n}\right)}(1+t-b)^{1-\operatorname{sgn} M} d b
$$

for all $t>0$. Here we have set $M=\sqrt{m^{2}-\frac{n^{2}}{4}}$.

Here, $W^{k, p}\left(\mathbb{R}^{n}\right)=\left\{u \in L^{p}\left(\mathbb{R}^{n}\right): D^{\alpha} u \in L^{p}\left(\mathbb{R}^{n}\right),|\alpha| \leq k\right\}$, denotes a Sobolev space with the norm

$$
\begin{aligned}
\|u\|_{W^{k, p}\left(\mathbb{R}^{n}\right)} & =\left(\sum_{|\alpha| \leq k} \int_{\mathbb{R}^{n}}\left|D^{\alpha} u\right|^{p}\right)^{1 / p}, \quad(1 \leq p<\infty), \\
\|u\|_{W^{k, \infty}\left(\mathbb{R}^{n}\right)} & =\sum_{|\alpha| \leq k} \operatorname{ess} \sup _{\mathbb{R}^{n}}\left|D^{\alpha} u\right| .
\end{aligned}
$$

\section{Preliminary}

Throughout this paper, the positive constants which may change, are denoted by the same letters $C$. We prepare the inequality for proving Theorem 2 . First of all, we introduce the hypergeometric function $F(a, b ; c ; \zeta)$ and study its property. It is defined by the power series

$$
F(a, b ; c ; \zeta)=\sum_{n=0}^{\infty} \frac{(a)_{n}(b)_{n}}{(c)_{n}} \frac{\zeta^{n}}{n !}, \quad|\zeta|<1,
$$

where $a, b, c \in \mathbb{C}$ with $c \neq 0,-1,-2, \ldots$, and we denoted

$$
\left\{\begin{array}{l}
(a)_{0}=1, \\
(a)_{n}=\Gamma(a+n) / \Gamma(a)=a(a+1) \ldots(a+n-1), \quad n=1,2,3, \ldots
\end{array}\right.
$$

Here $\Gamma$ is the gamma function (see e.g. [1]).

Lemma 1. Assume that $M \geq 0$. Then, for $z>1$,

$$
\int_{0}^{z-1}\left((z+1)^{2}-y^{2}\right)^{-\frac{1}{2}}\left|F\left(\frac{1}{2}+i M, \frac{1}{2}+i M ; 1 ; \frac{(z-1)^{2}-y^{2}}{(z+1)^{2}-y^{2}}\right)\right| d y \leq C(1+\ln z)^{1-\operatorname{sgn} M}(z-1)(z+1)^{-1} .
$$

Proof. First, we consider $M>0$. From the formulas 15.3.6 and 15.3.10 of chapter 15 in [1], we get

$$
\left|F\left(\frac{1}{2}+i M, \frac{1}{2}+i M ; 1 ; \xi\right)\right| \leq C,
$$


for all $\xi \in[0,1)$. Hence we have

$$
\begin{aligned}
\int_{0}^{z-1}\left((z+1)^{2}-y^{2}\right)^{-\frac{1}{2}}\left|F\left(\frac{1}{2}+i M, \frac{1}{2}+i M ; 1 ; \frac{(z-1)^{2}-y^{2}}{(z+1)^{2}-y^{2}}\right)\right| d y & \leq C \int_{0}^{z-1}\left((z+1)^{2}-y^{2}\right)^{-\frac{1}{2}} d y \\
& \leq C(z-1)(z+1)^{-1}
\end{aligned}
$$

Next, we consider $M=0$. If $1<z<N$ with some constant $\mathrm{N}$, then the argument of hypergeometric function is bounded,

$$
\frac{(z-1)^{2}-y^{2}}{(z+1)^{2}-y^{2}} \leq \frac{(z-1)^{2}}{(z+1)^{2}} \leq \frac{(N-1)^{2}}{(N+1)^{2}} \leq C
$$

for all $y \in(0, z-1)$, and

$$
\begin{aligned}
\int_{0}^{z-1}\left((z+1)^{2}-y^{2}\right)^{-\frac{1}{2}}\left|F\left(\frac{1}{2}, \frac{1}{2} ; 1 ; \frac{(z-1)^{2}-y^{2}}{(z+1)^{2}-y^{2}}\right)\right| d y & \leq C \int_{0}^{z-1}\left((z+1)^{2}-y^{2}\right)^{-\frac{1}{2}} d y \\
& \leq C(z-1)(z+1)^{-1}
\end{aligned}
$$

Then, we consider the case $z \geq N$. In particular, we choose $N>6$ and split the integral into two parts:

$$
\begin{aligned}
& \int_{0}^{z-1}\left((z+1)^{2}-y^{2}\right)^{-\frac{1}{2}}\left|F\left(\frac{1}{2}, \frac{1}{2} ; 1 ; \frac{(z-1)^{2}-y^{2}}{(z+1)^{2}-y^{2}}\right)\right| d y \\
& =\int_{0}^{\sqrt{(z+1)^{2}-8 z}}\left((z+1)^{2}-y^{2}\right)^{-\frac{1}{2}}\left|F\left(\frac{1}{2}, \frac{1}{2} ; 1 ; 1-\frac{4 z}{(z+1)^{2}-y^{2}}\right)\right| d y \\
& \quad+\int_{\sqrt{(z+1)^{2}-8 z}}^{z-1}\left((z+1)^{2}-y^{2}\right)^{-\frac{1}{2}}\left|F\left(\frac{1}{2}, \frac{1}{2} ; 1 ; 1-\frac{4 z}{(z+1)^{2}-y^{2}}\right)\right| d y
\end{aligned}
$$

For the second integral, if $y \geq \sqrt{(z+1)^{2}-8 z}$, then

$$
\frac{4 z}{(z+1)^{2}-y^{2}} \geq \frac{1}{2} \Rightarrow 0<1-\frac{4 z}{(z+1)^{2}-y^{2}} \leq \frac{1}{2}
$$

implies

$$
\left|F\left(\frac{1}{2}, \frac{1}{2} ; 1 ; 1-\frac{4 z}{(z+1)^{2}-y^{2}}\right)\right| \leq C
$$

Hence, we get

$$
\begin{aligned}
\int_{\sqrt{(z+1)^{2}-8 z}}^{z-1}\left((z+1)^{2}-y^{2}\right)^{-\frac{1}{2}}\left|F\left(\frac{1}{2}, \frac{1}{2} ; 1 ; 1-\frac{4 z}{(z+1)^{2}-y^{2}}\right)\right| d y & \leq C \int_{0}^{z-1}\left((z+1)^{2}-y^{2}\right)^{-\frac{1}{2}} d y \\
& \leq C(z-1)(z+1)^{-1}
\end{aligned}
$$

For the first integral, $y \leq \sqrt{(z+1)^{2}-8 z}$ and $z \geq N \geq 6$ imply $8 z \leq(z+1)^{2}-y^{2}$. It follows

$$
\left|F\left(\frac{1}{2}, \frac{1}{2} ; 1 ; 1-\frac{4 z}{(z+1)^{2}-y^{2}}\right)\right| \leq C\left|\ln \left(\frac{4 z}{(z+1)^{2}-y^{2}}\right)\right| \leq C(1+\ln z) .
$$


Hence, we get

$$
\begin{aligned}
\int_{0}^{\sqrt{(z+1)^{2}-8 z}}\left((z+1)^{2}-y^{2}\right)^{-\frac{1}{2}}\left|F\left(\frac{1}{2}, \frac{1}{2} ; 1 ; 1-\frac{4 z}{(z+1)^{2}-y^{2}}\right)\right| d y & \leq C(1+\ln z) \int_{0}^{z-1}\left((z+1)^{2}-y^{2}\right)^{-\frac{1}{2}} d y \\
& \leq C(1+\ln z)(z-1)(z+1)^{-1}
\end{aligned}
$$

This completes the proof.

\section{Fundamental solution of the Klein-Gordon equation}

It was shown in [8] that the solution $u=u(x, t)$ of the initial value problem

$$
u_{t t}-e^{2 t} \Delta u+M^{2} u=f, \quad u(x, 0)=0, \quad u_{t}(x, 0)=0, \quad(x, t) \in \mathbb{R}^{n} \times(0, \infty),
$$

with $f \in C^{\infty}\left(\mathbb{R}^{n}\right)$ is given by

$$
u(x, t)=2 \int_{0}^{t} d b \int_{0}^{e^{t}-e^{b}} d r v(x, r ; b)\left(4 e^{b+t}\right)^{i M}\left(\left(e^{t}+e^{b}\right)^{2}-r^{2}\right)^{-\frac{1}{2}-i M} F\left(\frac{1}{2}+i M, \frac{1}{2}+i M ; 1 ; \frac{\left(e^{b}-e^{t}\right)^{2}-r^{2}}{\left(e^{b}+e^{t}\right)^{2}-r^{2}}\right)
$$

where $v(x, t ; b)$ is the solution to the following initial value problem for the wave equation

$$
v_{t t}-\Delta v=0, \quad v(x, 0 ; b)=f(x, b), \quad v_{t}(x, 0 ; b)=0, \quad(x, t) \in \mathbb{R}^{n} \times(0, \infty),
$$

where $b>0$.

\section{Proof of theorem 2}

The solution $v(x, t)$ of the initial value problem (12) satisfies

$$
\|v(., r ; b)\|_{L^{\infty}\left(\mathbb{R}^{n}\right)} \leq C(1+r)^{-\frac{n-1}{2}}\|f(., b)\|_{W^{[n / 2]+1,1}\left(\mathbb{R}^{n}\right)},
$$

for all $r>0$, if $n \geq 2$ (see e.g. [10]). By using (13), we have

$$
\begin{aligned}
\|u(., t)\|_{L^{\infty}\left(\mathbb{R}^{n}\right) \leq} & C \int_{0}^{t}\|f(., b)\|_{W^{[n / 2]+1,1}\left(\mathbb{R}^{n}\right)} d b \\
& \times \int_{0}^{e^{t}-e^{b}}(1+r)^{-\frac{n-1}{2}}\left(\left(e^{t}+e^{b}\right)^{2}-r^{2}\right)^{-\frac{1}{2}}\left|F\left(\frac{1}{2}+i M, \frac{1}{2}+i M ; 1 ; \frac{\left(e^{b}-e^{t}\right)^{2}-r^{2}}{\left(e^{b}+e^{t}\right)^{2}-r^{2}}\right)\right| d r \\
\leq & C \int_{0}^{t}\|f(., b)\|_{W^{[n / 2]+1,1}\left(\mathbb{R}^{n}\right)} d b \\
& \times \int_{0}^{e^{t}-e^{b}}\left(\left(e^{t}+e^{b}\right)^{2}-r^{2}\right)^{-\frac{1}{2}}\left|F\left(\frac{1}{2}+i M, \frac{1}{2}+i M ; 1 ; \frac{\left(e^{b}-e^{t}\right)^{2}-r^{2}}{\left(e^{b}+e^{t}\right)^{2}-r^{2}}\right)\right| d r .
\end{aligned}
$$

If we change the variable by $r=e^{b} y$, then we obtain

$$
\begin{aligned}
\|u(., t)\|_{L^{\infty}\left(\mathbb{R}^{n}\right)} \leq & C \int_{0}^{t}\|f(., b)\|_{W^{[n / 2]+1,1}\left(\mathbb{R}^{n}\right)} d b \\
& \times \int_{0}^{e^{t-b}-1}\left(\left(e^{t-b}+1\right)^{2}-y^{2}\right)^{-\frac{1}{2}}\left|F\left(\frac{1}{2}+i M, \frac{1}{2}+i M ; 1 ; \frac{\left(e^{t-b}-1\right)^{2}-y^{2}}{\left(e^{t-b}+1\right)^{2}-y^{2}}\right)\right| d y .
\end{aligned}
$$


Then, we apply Lemma 1 with $z=e^{t-b}$ to the second integrand of the right-hand side of the inequality (14);

$$
\|u(., t)\|_{L^{\infty}\left(\mathbb{R}^{n}\right)} \leq C \int_{0}^{t}\|f(., b)\|_{W^{[n / 2]+1,1}\left(\mathbb{R}^{n}\right)}\left(e^{t-b}-1\right)\left(e^{t-b}+1\right)^{-1}(1+t-b)^{1-\operatorname{sgn} M} d b,
$$

and we obtain (8). This completes the proof of Theorem 2.

\section{Competing interests}

The authors declare that they have no competing interests.

\section{Authors' contributions}

All authors have contributed to all parts of the article. All authors read and approved the final manuscript.

\section{References}

[1] H. Bateman, A. Erdelyi, "Higher Transcendental Functions", 1,2, McGraw-Hill, New York, 1953.

[2] P. Brenner, On the existence of global smooth solutions of certain semilinear hyperbolic equations, Math. Z. 167 (2) (1979), 99-135.

[3] J. Ginibre, G. Velo, The global Cauchy problem for the nonlinear Klein-Gordon equation, Math Z. 189(4) (1985), 487-505.

[4] J. Ginibre, G. Velo, The global Cauchy problem for the nonlinear Klein-Gordon equation II, Ann. Inst. H. Poincarè Anal. Linèaire 6(1) (1989), 15-35.

[5] K. Jörgens, Das Anfangswertproblem im Grossen für eine Klasse nichtlinearer Wellengleichungen, Math. Z. 77 (1961), $295-308$.

[6] C. Møller, “The Theory of Relativity”, Clarendon Press, Oxford, 1972.

[7] H. Pecher, $L^{p}$-Abschützungen und klassische Lösungen für nichtlineare Wellengleichungen. I, Math. Z. 150 (1976), $159-183$.

[8] K. Yagdjian, A. Galstian, The Klein-Gordon equation in anti-de Sitter spacetime, Rend. Sem. Mat. Univ. Pol. Torino 67 (2) (2009), 271-292.

[9] A. Galstian, $L^{p}-L^{q}$ Decay estimates for the Klein-Gordon equation in anti-de Sitter space-time, Rend. Istit. Mat. Univ. Trieste 42 (2010), 27-50.

[10] W. von Wahl, $L^{p}$-decay rates for homogeneous wave-equations, Math. Z. 120 (1971), 93-106. 